\title{
Primary pulmonary blastoma of monophasic variety- diagnosis and management
}

\author{
Jitendra H Mistry', Sumita B Pawar ${ }^{2}$, Harshad Mehta ${ }^{1}$, Aron Frederik Popov ${ }^{3,4,5^{*}}$ and Prashant Nanasaheb Mohite ${ }^{1,4}$
}

\begin{abstract}
Pulmonary blastoma is a rare primary lung neoplasm, in that monophasic variety is far too rare. There are no specific clinical features seen for pulmonary blastoma; computed tomography and histopathology are diagnostic. Surgical excision is the treatment of choice; however, adjuvant chemotherapy and radiotherapy may be required in large and aggressive tumors.
\end{abstract}

Keywords: Pulmonary blastoma, Lung cancer

\section{Background}

Pulmonary blastomas are relatively rare group of primary lung neoplasms with disputed histogenesis and variable biologic behavior that are composed of immature malignant epithelial and/or mesenchymal tissues whose features may resemble early embryological lung tissues. Since the first report by Barrett \& Barnard, in 1945, many more cases of pulmonary blastoma have been reported [1]. Although over a hundred cases of pulmonary blastoma are reported in the literature, the monophasic variety is extremely rare [1,2]. Pulmonary blastoma is observed in the fourth decade of life with mean age of occurrence in adults being 43 years, and shows a strong female preponderance [2]. We present a case of a teenager affected with monophasic variety of pulmonary blastoma.

\section{Case presentation}

A young healthy female of 18 years presented with left sided chest pain not related to exertion since one month. Examination of respiratory system revealed decreased air entry on left side over mammary and supra-mammary regions. Chest X-ray showed about $10 \mathrm{~cm}$ sized rounded opacity on left upper quadrant (See Figure 1). Spiral computed tomography (CT) scan of chest confirmed the large, neoplastic mass with few necrotic and calcific foci

\footnotetext{
* Correspondence: Popov@med.uni-goettingen.de

${ }^{3}$ University of Göttingen, Göttingen, Germany

${ }^{4}$ Department of Cardiothoracic Transplantation \& Mechanical Support, Royal Brompton and Harefield NHS Trust, Harefield Hospital, Harefield, Middlesex UB9 6JH, UK

Full list of author information is available at the end of the article
}

involving left upper lobe (See Figure 2). Ultrasonography of the abdomen and pelvis showed no abnormality. CT guided biopsy was done and the smear showed the formation of tubules lined by columnar cells that contained PAS positive clear to pale eosinophilic cytoplasm with intervening area containing spindle cells suggestive of pulmonary blastoma (See Figure 3). The samples of biopsy were also sent for immunohistochemistry which showed the lining epithelial cells of tubules strongly expressing cytokeratin and the spindle cells expressing vimetin. The focal expression of desmin was also noticed.

Left postero-lateral thoracotomy through fifth intercostal space was done. Approximately $15 \times 15 \mathrm{~cm}$ sized mass occupying entire left upper lobe (See Figure 4) not involving parietal pleura was noticed. The left upper lobe along with the tumor was reflected anteriorly and inferiorly and the left pulmonary artery was exposed by opening the posterior mediastinal pleura. An artery to the posterior segment, the apical-posterior arteries and an artery to the anterior segment were encountered and divided between ligatures. Distal dissection over the pulmonary artery led to the lingular arteries, which were also divided in ligatures. The anterior and posterior portions of the fissure were cut opened with the fine dissection and the parenchymal surface on the lower lobe was sutured with Polypropylene 3-0 suture utilizing continuous technique. The upper lobe bronchus was visualized clearly and closed with the $30 \mathrm{~mm}$ linear (TA) stapler. The distal branches of the superior pulmonary vein were ligated and transected and the lobe along with the tumor 


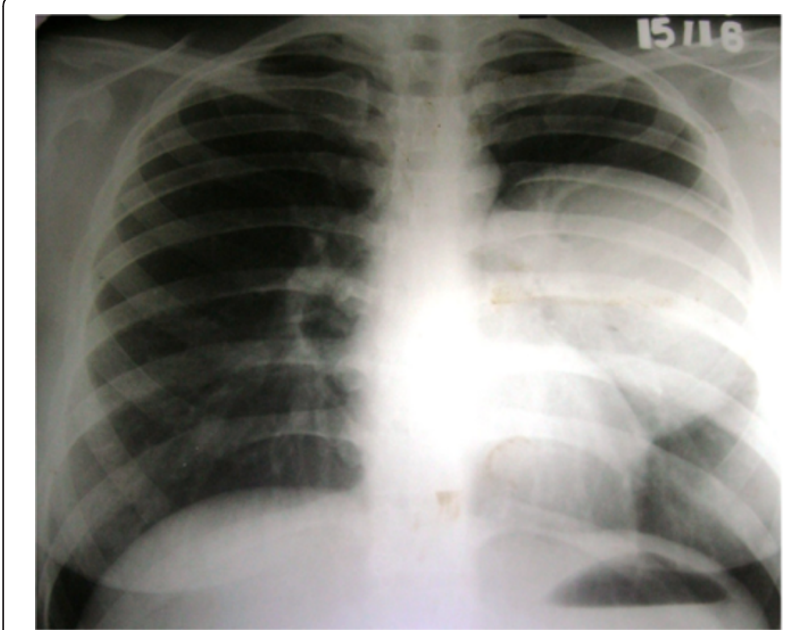

Figure $1 \mathrm{X}$ - ray chest showing soft tissue opacity in left upper lobe.

was removed (See Figure 5). Hilar lymph node sampling was performed, hemostasis achieved and the chest was closed routinely. The histopathology of the specimen was suggestive of well differentiated fetal adenocarcinoma (monophasic pulmonary blastoma) with clear margins without any evidence of tumor cells in lymph nodes. Post operative course was uneventful. Patient was given six cycles of Cisplatin based adjuvant chemotherapy and there was no evidence of recurrence or distant metastasis in two years of follow up.

\section{Discussion}

Pulmonary tumors of embryonic origin are rare and among them, pulmonary blastomas are probably the most uncommon [3]. They form about 0.25 to $0.50 \%$ of all lung neoplasm, presumably arising from primitive pulmonary mesenchyme and histologically they resemble fetal lung [1]. They tend to occur peripherally and have a poor prognosis. First described by Barnard in 1952, they have been divided into three subgroups viz. classic pulmonary blastoma, well-differentiated fetal adenocarcinoma also

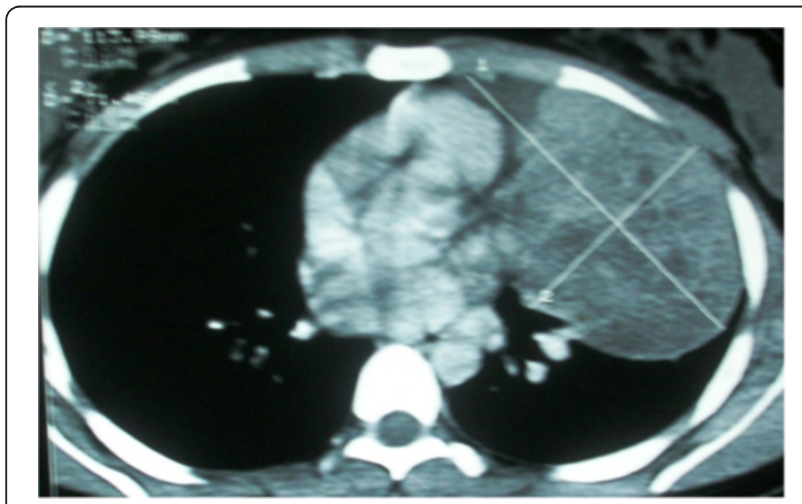

Figure 2 CT chest showing tumor in left upper lobe.

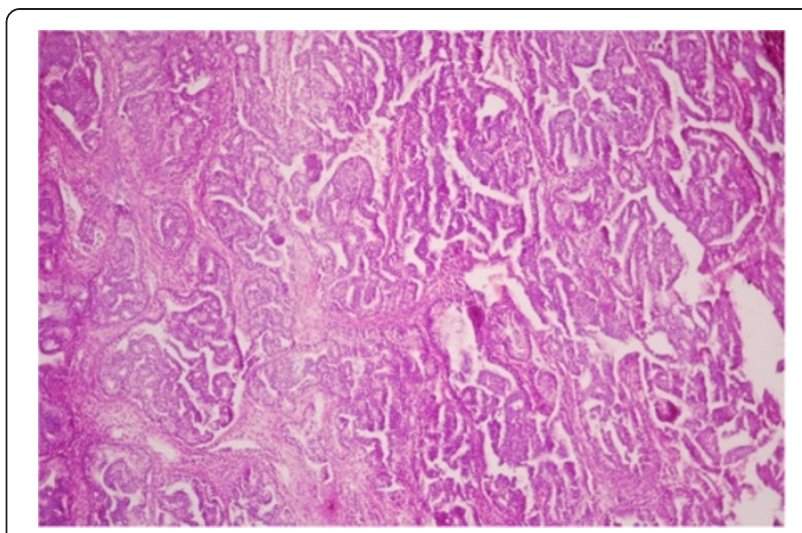

Figure 3 Histopathological picture.

called monophasic pulmonary blastoma and pleuropulmonary blastoma [1]. Classical pulmonary blastoma is the most common of these three subtypes. Some authors have classified the pulmonary blastoma into biphasic and Monophasic variety [4]. Biphasic pulmonary blastomas contain both neoplastic glandular tissue and either adult sarcomatous or embryonic mesenchymal tissue whereas Monophasic blastomas contain solely malignant glands of embryonic appearance [5]. A pulmonary blastoma should not be confused with the pleuropulmonary blastoma of childhood, which is analogous to Wilm's tumor of the lung in which there is no recognizable neoplastic epithelium.

Approximately $25 \%$ to $40 \%$ of patients are asymptomatic at presentation, with incidental diagnosis by chest radiography. Common symptoms are cough, hemoptysis and chest pain while pleural effusion is seen unusually. Present case was having chest pain as a main complaint. The majority of pulmonary blastomas have been reported in adults, occurs mainly in young women [2] and is associated with smoking in $82 \%$ cases [1]. The monophasic type presents better prognosis compared to biphasic variety.

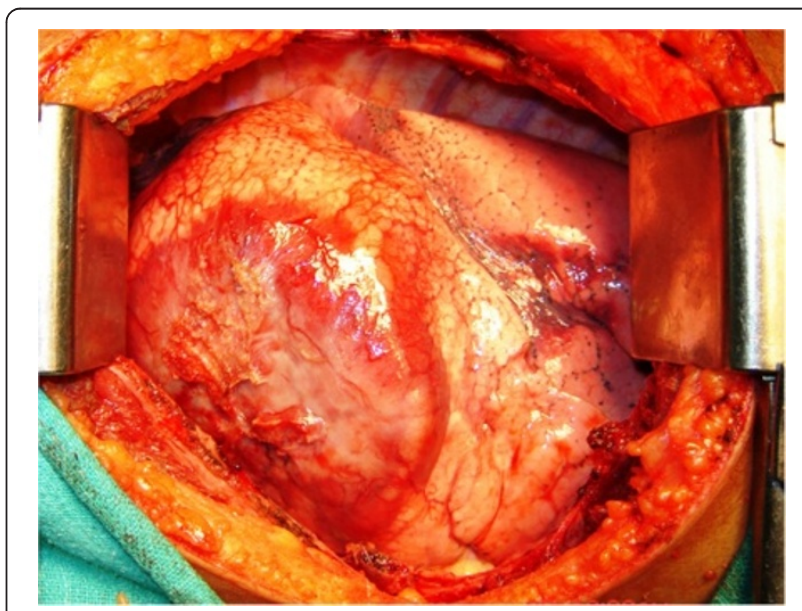

Figure 4 Left thoracotomy showing tumor in upper lobe. 


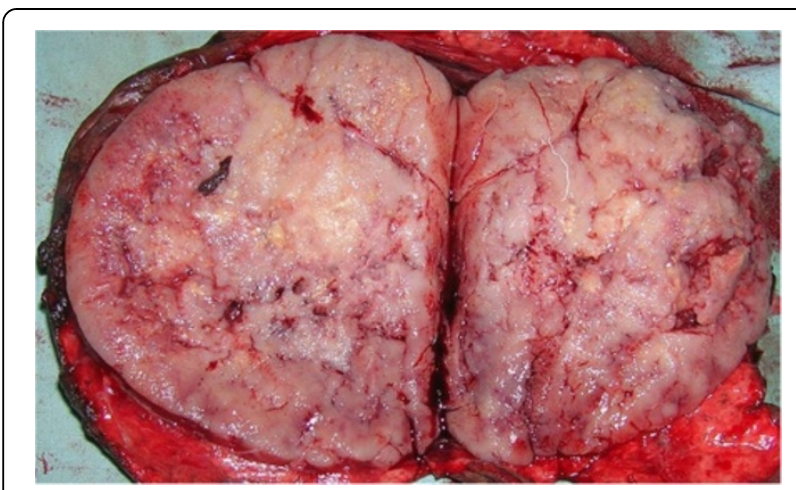

Figure 5 Bisected specimen with lingula.

Radiography shows a well-demarcated peripheral lesion, well defined homogenous peripherally placed radiopacities with the lesions ranging from 2.5 to $25 \mathrm{~cm}$ $[1,6]$. On ultrasound examination, they shows heterogeneous appearance with solid and few cystic areas which indicate necrotic component. On computed tomography, heterogeneous appearance with typically enhancing whorls of solid tissue and no enhancing areas of necrosis are seen [6]. Metastases to same or opposite lung as well as involvement of mediastinum and adjacent chest wall can also be seen.

On gross pathology, the tumor is rounded, well circumscribed. In some, it may show lobulated appearance and multiple lesions. On immunohistochemistry expression of cytokeratin and vimentin is seen in the blastomatous component, the epithelial elements in blastomas react positively for keratin, carcinoembryonic antigen, epithelial membrane antigen, and milk fat globulin [7]. In the present case the tumor expressed cytokeratin, vimentin and keratin.

Surgery is the preferred treatment and is mandatory [8] whenever possible, often with adjuvant chemotherapy and/or radiotherapy [9]. Some authors suggest that the combination of surgery, adjuvant radiotherapy and chemotherapy based on cisplatin and etoposide should be considered [9]. Using an adjuvant protocol similar to the one used in the treatment of germ cell tumors (cisplatin, VP-16, uromitexan, ifosfamide and 64 Gy of mediastinal radiotherapy), a Swiss group has reported a 33-month survival in a stage III-A patient (pT3N2M0) [2]. In most health care facilities, radiotherapy is used to treat cases that do not respond to other forms of treatment [10]. Prognosis is worse if the tumour is larger than $5 \mathrm{~cm}$ at presentation [9]. Distant metastases are frequently seen in the liver, central nervous system, mediastinum, and bones [7]. This case was presented with $15 \times 15$ centimeters with no evidence of distant metastasis.

Overall, survival is $25 \%$ at 1 year and $16 \%$ at 5 years. Factors that indicate a poor prognosis are tumour recurrence, metastases at initial presentation, tumor greater than $5 \mathrm{~cm}$, and lymph node metastases [1]. The prognosis is worse with the biphasic than monophasic type blastoma because of the high incidence of metastasis in the former $[1,5]$. No therapeutic guidelines exist. If metastases are present, survival is unlikely despite therapy.

\section{Conclusions}

Monophasic pulmonary blastomas are rare neoplasms with good overall prognosis. Surgical excision is the treatment of choice and produce better prognosis with adjuvant chemotherapy.

\section{Consent}

Written informed consent was obtained from the patients for publication of this Case report and accompanying images. A copy of the written consent is available for review by the Editor-in-Chief of this journal.

\section{Abbreviations \\ CT scan: Computed tomogram.}

\section{Competing interests}

The authors declare that they have no competing interests.

\section{Authors' contributions}

JHM drafted the manuscript, SBP analyzed and interpreted the patient data, $\mathrm{HM}$ performed the performed the surgery and was a major contributor in writing the manuscript, AFP conceptualize the manuscript and PNM involved in the crucial revisions of manuscript. All authors read and approved the final manuscript.

\section{Acknowledgements}

None of the authors have any external funding source.

\section{Author details}

${ }^{1}$ Department of Cardiothoracic Surgery, Medical College, Vadodara, Gujarat, India. ${ }^{2}$ Department of Pathology, ACPM Medical College, Dhule, India.

${ }^{3}$ University of Göttingen, Göttingen, Germany. ${ }^{4}$ Department of Cardiothoracic Transplantation \& Mechanical Support, Royal Brompton and Harefield NHS

Trust, Harefield Hospital, Harefield, Middlesex UB9 6JH, UK.

${ }^{5}$ Georg-August-University Goettingen, Robert-Koch-Str. 40, D - 37075 , Goettingen, Germany.

Received: 17 March 2013 Accepted: 6 June 2013

Published: 7 June 2013

\section{References}

1. Koss MN, Hochholzer L, O'Leary T: Pulmonary blastomas. Cancer 1991, 67:2368-2381.

2. Robert J, Pache JC, Seium Y, De Perrot M, Spiliopoulos A: Pulmonary blastoma: report of five cases and identification of clinical features suggestive of the disease. Eur J Cardiothorac Surg 2002, 22(5):708-711.

3. Kliem V, Bugge M, Leimenstoll K, Maschek H: Pulmonary blastoma a rare tumour. Clin Investig 1992, 70:927-931.

4. Muller-Hermelink HK, Kaiserling E: Pulmonary adenocarcinoma of fetal type: alternating differentiation argues in favour of a common endodermal stem cell. Virchows Arch A Pathol Anat Histopathol 1986, 409:195-210.

5. Hage E: Electron microscopic identification of endocrine cells in the bronchial epithelium of human foetuses. Acta Pathol Microbiol Scand 1972, 80:143-144.

6. Armstrong P: Neoplasms of the lungs, airways and pleura. In Imaging of diseases of the chest; 2nd ed. Edited by Armstrong P, Wilson AG, Dee P, Hansell DM. Mosby: Year Book; 1995:315.

7. Barson AJ, Jones AW, Lodge KV: Pulmonary blastoma. J Clin Pathol 1968, 21:480-485 
8. Zaidi A, Zamvar V, Macbeth F, Gibbs AR, Kulatilake N, Butchart EG:

Pulmonary blastoma: medium-term results from a regional center. Ann Thorac Surg 2002, 73(5):1572-1575.

9. Cutler CS, Michel RP, Yassa M, Langleben A: Pulmonary blastoma: a case report of a patient with a 7-year remission and review of chemotherapy experience in the world literature. Cancer 1998, 82:462-467.

10. Surmont VF, van Klaveren RJ, Nowak PJ, Zondervan PE, Hoogsteden $H C$, van Meerbeeck JP: Unexpected response of a pulmonary blastoma on radiotherapy: case report and review of the literature. Lung Cancer 2002, 36(2):207-211.

doi:10.1186/1749-8090-8-144

Cite this article as: Mistry et al.: Primary pulmonary blastoma of monophasic variety- diagnosis and management. Journal of Cardiothoracic Surgery 2013 8:144.

\section{Submit your next manuscript to BioMed Central and take full advantage of:}

- Convenient online submission

- Thorough peer review

- No space constraints or color figure charges

- Immediate publication on acceptance

- Inclusion in PubMed, CAS, Scopus and Google Scholar

- Research which is freely available for redistribution 